\title{
SCIENTIFIC REPORTS

\section{OPEN Iron acquisition in Pseudomonas aeruginosa by the siderophore pyoverdine: an intricate interacting network including periplasmic and membrane proteins}

\begin{abstract}
Anne Bonneau ${ }^{1,2}$, Béatrice Roche ${ }^{1,2^{*}}$ \& Isabelle J. Schalk $\mathbb{1}^{1,2^{*}}$
Pyoverdine (PVDI) has been reported to act both as a siderophore for scavenging iron (a key nutrient) and a signaling molecule for the expression of virulence factors. This compound is itself part of a core set of virulence factors produced by Pseudomonas aeruginosa during infections. Once secreted into the bacterial environment and having scavenged ferric iron, $\mathrm{PVDI}-\mathrm{Fe}^{3+}$ is taken back into the $P$. aeruginos $a$ periplasm via the outer membrane transporters FpvAI and FpvB. Iron release from PVDI in the bacterial periplasm involves numerous proteins encoded by the fpvGHJKCDEF genes and a mechanism of iron reduction. Here, we investigated the global interacting network between these various proteins using systematic bacterial two-hybrid screening. We deciphered a network of five interacting proteins composed of two inner-membrane proteins, FpvG (iron reductase) and FpvH (unknown function), and three periplasmic proteins, FpvJ (unknown function), FpvF (periplasmic PVDI-binding protein), and $\mathrm{FpvC}$ (iron periplasmic-binding protein). This interacting network strongly suggests the existence of a large protein machinery composed of these five proteins, all playing a role in iron acquisition by PVDI. Furthermore, we discovered an interaction between the periplasmic siderophore binding protein FpvF and the PvdRT-OpmO efflux pump, also suggesting a role for FpvF in apo-PVDI recycling and secretion after iron delivery. These results highlight a multi-protein complex that drives iron release from PVDI in the periplasm of $P$. aeruginosa.
\end{abstract}

Pseudomonas aeruginosa is an opportunistic human Gram-negative pathogen considered by the World Health Organization to be an antibiotic-resistant priority pathogen ${ }^{1,2}$. During infections, P. aeruginosa faces a stressful environment and must overcome host-defense mechanisms. To survive within the host, $P$. aeruginosa secretes a large number of virulence factors, including the siderophores pyoverdines ${ }^{2,3}$. Siderophores are small organic compounds produced and secreted by bacteria to access iron ${ }^{4}$, a key nutrient essential for bacterial growth and virulence. Strains unable to produce pyoverdines have been reported to exhibit reduced virulence during infections in mice ${ }^{5}$. The role of pyoverdines in the virulence of $P$. aeruginos $a$ has also been ascertained using rabbit and mouse lung infection models ${ }^{6-8}$. Pyoverdines are reported to have a dual role during infection. They are used as a siderophore by $P$. aeruginosa to scavenge iron from the host protein $s^{5,8}$ and also acts as a signaling molecule for the production of two major virulence factors, exotoxin A and the endo-proteinase $\operatorname{PrpL}^{3,9}$.

More generally, all fluorescent Pseudomonas species produce specific pyoverdines as major siderophores to access iron. These pyoverdines are all composed of a peptide of 6 to 12 amino acids, with a specific sequence, and linked to a chromophore derived from 2,3-diamino-6,7-dihydroxyquinoline ${ }^{10}$. The sequence of the peptide moiety of the different pyoverdines is very different in amino acid composition and in length among pyoverdines and is a determinant specific of each pseudomonads species ${ }^{10-14}$. P. aeruginosa strains produce three distinct pyoverdine types (PVDI, PVDII and PVDIII) each characterized by a different peptide chain ${ }^{15}$ and PVDI is the siderophore produced by $P$. aeruginosa $\mathrm{PAO}$. Molecular mechanisms involved in iron acquisition by pyoverdines have mostly been investigated in P. aeruginosa PAO1.

${ }^{1}$ CNRS, UMR7242, ESBS, Illkirch, Strasbourg, France. ${ }^{2}$ Université de Strasbourg, UMR7242, ESBS, Illkirch, Strasbourg, France. *email: beatrice.roche@unibas.ch; schalk@unistra.fr 


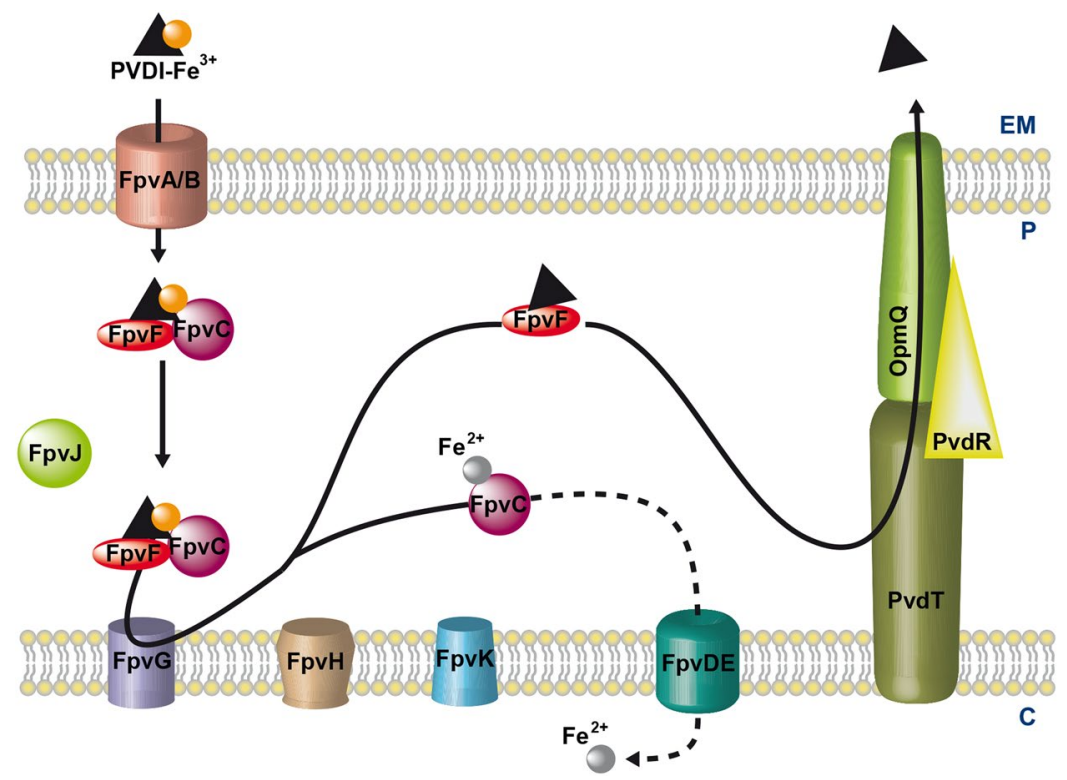

Figure 1. Model of $\mathrm{Fe}^{3+}$ uptake by the siderophore PVDI in P. aeruginosa. For details, see the Introduction section. EM: extracellular medium; P: periplasm; C: cytoplasm.

PVDI is synthesized by non-ribosomal peptide synthetases in the bacterial cytoplasm ${ }^{16,17}$ and then matures in the periplasm ${ }^{18}$ before secretion into the extracellular medium by the PvdRT-OpmQ ATP-dependent efflux pump $^{19}$. In the bacterial environment, PVDI chelates ferric iron, yielding the PVDI-Fe ${ }^{3+}$ complex ${ }^{20}$. Ferric complexes of this siderophore are then recognized at the bacterial surface and imported across the outer membrane by two specific TonB-dependent transporters, FpvAI and FpvB (Fig. 1), with the TonB-ExbB-ExbD inner-membrane protein complex providing the necessary energy $\mathrm{y}^{21-24}$. Once in the periplasm, $\mathrm{PVD}-\mathrm{Fe}^{3+}$ is bound by the two periplasmic proteins, FpvC and $\mathrm{FpvF}^{25}$. Iron release from PVDI occurs in the bacterial periplasm and involves no chemical modification of the siderophore but rather iron reduction by the FpvG inner-membrane reductase $\mathrm{e}^{26-28}$. $f p v G$ is localized next to $f p v H, f p v J$, and $f p v K$ genes encoding three proteins of unknown function, but of which expression is required for optimal activity of $\mathrm{FpvG}^{28}$. Sequence alignment of $\mathrm{FpvC}$ revealed that this protein belongs to a group of metal-binding periplasmic protein $s^{25}$, and previous in vitro studies of PVDI-Fe dissociation in the presence of DTT showed that FpvC can apparently bind ferrous iron after the reduction step and its dissociation from $\mathrm{PVDI}^{28}$. Iron is translocated further across the inner membrane into the cytoplasm by the predicted $\mathrm{ABC}$ transporter FpvDE ${ }^{25}$. All four proteins FpvC, FpvD, FpvE and FpvF, which genes are localized next to $f p v G H J K$ genes, are also necessary for efficient dissociation of iron from PVDI ${ }^{28}$. After iron release, the apo form of PVDI is recycled into the extracellular medium by the PvdRT-OpmQ efflux pump, with the ability to again chelate $\mathrm{Fe}^{3+}$ in the bacterial environment ${ }^{29,30}$. Dimers of the periplasmic protein FpvF are able to bind apo-PVDI ${ }^{25}$ and the recycling of apo-PVDI has been shown to be partially abolished in an $\Delta f p v F$ mutant ${ }^{28}$, suggesting a role of FpvF in apo-PVDI recycling. Although it has been shown that FpvC and FpvF are able to form a complex that binds PVD-Fe ${ }^{3+}, 25$, the overall interaction network between all the proteins encoded by the $f p v G H J K C D E F$ genes has not been yet investigated.

Here, we characterized the interacting network between $P$. aeruginosa proteins of the PVDI-Fe ${ }^{3+}$ uptake pathway using the bacterial E. coli adenylate cyclase two-hybrid system (BACTH) for high throughput interaction screening. The results of BACTH screening revealed (i) an interaction between the two inner-membrane proteins FpvG and FpvH, (ii) an FpvJ-FpvC-FpvF periplasmic complex, and (iii) the ability of the two inner-membrane proteins (FpvG and $\mathrm{FpvH}$ ) and the three periplasmic proteins (FpvC, FpvF, and FpvJ) to interact in a membrane machinery complex. These complexes were further confirmed by purification and pulldown experiments. We also found that the periplasmic apo-PVDI binding protein $\mathrm{FpvF}^{25}$ is able to interact with PvdT, the inner-membrane protein of the PvdRT-OpmQ efflux pump, suggesting a role for FpvF in the transport of apo-PVDI to PvdRT-OpmQ through the bacterial periplasm. Our study has allowed unprecedented deciphering of the interacting network of the various proteins involved in $\mathrm{Fe}^{3+}$ release from PVDI in the periplasm of P. aeruginosa, linking both membrane and periplasmic proteins.

\section{Results}

Interaction between FpvG and FpvH and formation of an inner-membrane complex. Previous studies have demonstrated that expression of the $\mathrm{FpvH}, \mathrm{FpvK}$, and FpvJ proteins is required for optimal reductase activity of $\mathrm{FpvG}^{28}$. Moreover, genes encoding the FpvG, FpvH, FpvJ, and FpvK proteins are organized in an operon $^{31}$ and it is well recognized that adjacent genes tend to encode interacting proteins ${ }^{32}$. FpvG, FpvH, and FpvK have been predicted to be inner-membrane proteins and FpvJ periplasmic, because of a signal peptide ${ }^{28}$. We deciphered the interacting network between the membrane proteins FpvG, FpvH, and FpvK by performing systematic BACTH screening in E. coli, which is based on the reconstitution of adenylate-cyclase activity. The 


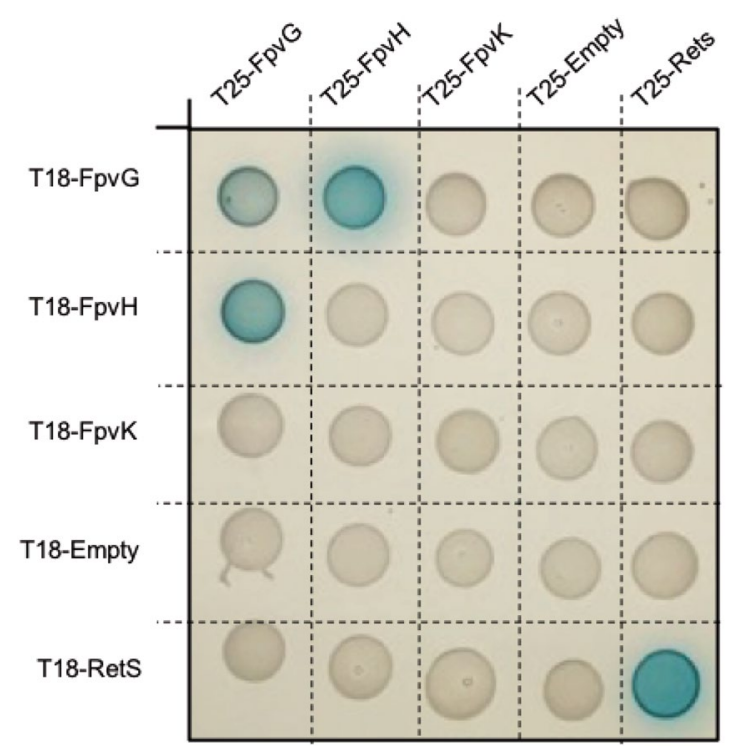

Figure 2. Interacting network with the membrane proteins FpvG, FpvH and FpvK. DHM1 cells producing the protein of interest fused to the T18 or T25 domain of adenylate cyclase were spotted on indicator plates containing X-gal for BATCH screening. Each protein was tested with an empty vector and the RetS protein, which is not related to the PVDI pathway. RetS is able to form dimers and serves as a positive control ${ }^{37}$. The blue color indicates an interaction between the two proteins of interest. The experiment was repeated three times with each time 10 colonies as described in Materials and Methods. A representative image is shown.
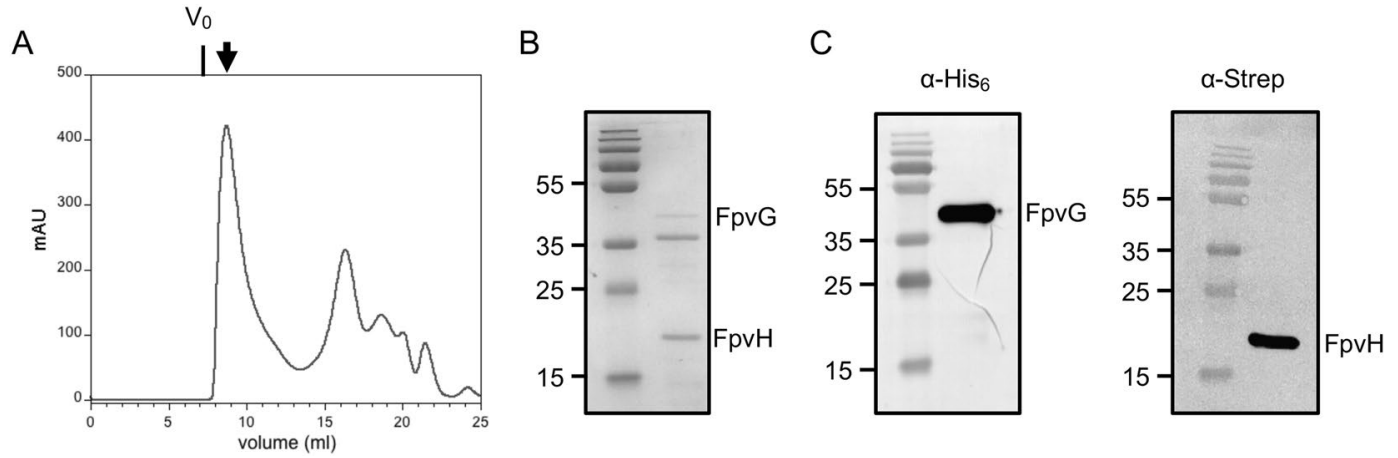

Figure 3. Purification of the inner-membrane FpvG-FpvH complex. (A) Size exclusion chromatography analysis of the solubilized and purified $\mathrm{FpvG}_{\mathrm{His} 6}-\mathrm{FpvH}_{\text {Strep }}$ complex on a Superdex 200 10/300 GL column. The dead volume is indicated as $\mathrm{V}_{0}$. The arrow shows the fraction containing the $\mathrm{FpvG}_{\mathrm{His} 6}-\mathrm{FpvH}_{\text {Strep }}$ complex. (B) SDS-PAGE of the purified complex analyzed by Coomassie blue staining. (C) Immunoblot analysis of the purified complex using specific anti-His and anti-Strep antibodies. The molecular mass markers $(\mathrm{kDa})$ are indicated on the left. The predicted size of $\mathrm{FpvG}_{\mathrm{His} 6}$ and $\mathrm{FpvH}_{\text {Strep }}$ are $46 \mathrm{kDa}$ and $21 \mathrm{kDa}$ respectively (Table S3 in Supplemental Material). Entire SDS-PAGE and blots are shown in Fig. S2 in Supplementary Information.

full-length $f p v G, f p v H$, and $f p v K$ genes were fused to the T25/T18 domains of adenylate cyclase in the two-hybrid vectors. Screening of the possible protein-protein interactions between FpvG, FpvH and FpvK on indicator plates containing X-gal highlighted an interaction between FpvG and FpvH (Fig. 2), whereas no interaction could be observed for FpvK. FpvG interacted with itself, suggesting at least dimerization of this protein (Fig. 2). As FpvG was already been demonstrated to be an inner-membrane protein ${ }^{28}$, we investigated the subcellular localization of FpvH. Cell fractionation experiments showed that FpvH is also an inner-membrane protein (Fig. S1A in Supplemental Material).

We expressed FpvG and FpvH proteins with C-terminal $\mathrm{His}_{6}$ and Strep-tag sequences, respectively, in E. coli to validate their interaction. Bacterial membranes were solubilized in detergent and the complex purified by Strep-trap affinity followed by size-exclusion chromatography (Fig. 3A). The presence of both FpvG and FpvH proteins in the elution peak was confirmed by Coomassie-blue staining and immunoblot analysis using specific anti-His ${ }_{6}$ and anti-Strep antibodies (Fig. 3B,C). Isolation of the FpvG-FpvH complex confirmed the BACTH results and revealed the ability of FpvG reductase and FpvH to interact and form an inner-membrane complex. 
Interaction between $\mathrm{FpvJ}, \mathrm{FpvC}$, and $\mathrm{FpvF}$ and formation of a periplasmic protein complex. FpvC and FpvF are the two periplasmic-binding proteins associated with the $\mathrm{ABC}$ transporter $\mathrm{FpvDE}^{25}$. Cell fractionation also demonstrated periplasmic localization of FpvJ (Fig. S1B in Supplemental Material). The presence of some FpvJ protein also in the cytoplasmic fraction was probably due to newly synthesized FpvJ, as previously described for periplasmic proteins ${ }^{33}$. Since the indicator plates assay cannot be used to detect interactions between periplasmic proteins, we screened the interactions between FpvJ, FpvC, and FpvF by quantifying ß-galactosidase in liquid cultures (Fig. 4). Concerning the interaction between the three periplasmic proteins, we detected interactions between FpvJ and FpvC (Fig. 4A), FpvF with FpvJ and FpvC (Fig. 4B) as previously shown by Brillet et al. ${ }^{25}$, as well as an interaction of FpvC with itself (Fig. 4C). Altogether, our BACTH results show that the three proteins FpvC, FpvF, and FpvJ are able to all interact with each other, suggesting the existence of a periplasmic complex composed of the three proteins.

We overproduced FpvJ, FpvF, and FpvC in E. coli, adding a $\mathrm{His}_{6}$, Flag tag, and $\mathrm{HA}$ at the C-terminus of each protein, respectively, to confirm the existence of this periplasmic complex. The periplasmic fraction was incubated with anti-His beads. $\mathrm{FpvF}_{\mathrm{Flag}}$ and $\mathrm{FpvC}_{\mathrm{HA}}$ co-precipitated with $\mathrm{FpvJ}_{\mathrm{His} 6}$ (Fig. 5). Negative controls showed that neither $\mathrm{FpvF}_{\mathrm{Flag}}$ nor $\mathrm{FpvC}_{\mathrm{HA}}$ alone were retained on the anti-His beads. Overall, these results show that $\mathrm{FpvJ}$, $\mathrm{FpvC}$ and $\mathrm{FpvF}$ can form a periplasmic complex.

Interaction between the inner-membrane proteins FpvG and FpvH with the periplasmic proteins FpvJ, FpvC, and FpvF. Finally, we also tested the interactions between the inner-membrane and periplasmic proteins. BACTH analysis showed that both FpvG and FpvH membrane proteins interact with the three periplasmic proteins FpvJ, FpvF, and FpvC (Fig. 4). As with the membrane proteins, none of the periplasmic proteins interacted with FpvK in this two-hybrid approach (Fig. 4). Overall, BACTH screening revealed the existence of an interaction network between FpvG, FpvH, FpvJ, FpvF, and FpvC.

We next attempted to isolate all five proteins by pulldown experiments using an anti-Flag resin. The periplasmic fraction of E. coli overproducing $\mathrm{FpvJ}_{\mathrm{His}}, \mathrm{FpvC}_{\mathrm{HA}}$, and $\mathrm{FpvF}_{\mathrm{Flag}}$ was incubated with solubilized membranes containing $\mathrm{FpvG}_{\mathrm{His} 6}$ and $\mathrm{FpvH}_{\text {trrep }}$, and the mixture incubated with an anti-Flag resin. $\mathrm{FpvG}_{\mathrm{His}}, \mathrm{FpvJ}_{\mathrm{His} 6}$, and $\mathrm{FpvC}_{\mathrm{HA}}$ co-precipitated with $\mathrm{FpvF}_{\mathrm{Flag}}$ (Fig. 6). Equivalent results were obtained when fractions were incubated with PVDI-Fe (Fig. S5 in Supplemental Material). None of the non-Flag-tagged proteins were retained on the anti-Flag resin when incubated alone (Fig. S6 in Supplemental Material). We were unable to detect $\mathrm{FpvH}_{\text {Strep }}$ either due to immunodetection problems or because this protein is not present in the complex. Overall, these results confirm the existence of at least a four-protein complex, linking the inner-membrane FpvG protein and three periplasmic components of the PVDI pathway. In addition, this complex could be isolated in the presence or absence of PVDI-Fe.

FpvF interacts with the membrane protein PvdT of the PvdRTOpmQ efflux pump. Previous studies have shown that FpvF can form dimers that bind apo-PVDI ${ }^{25}$ and that PVDI recycling is altered in a $\Delta f p v F$ mutant ${ }^{28}$. Based on these observations, it seemed possible that FpvF is involved in PVDI recycling by interacting with proteins of the efflux pump PvdRT-OpmQ. Indeed, our BACTH analysis revealed an interaction between FpvF and the membrane protein PvdT (Fig. 7).

\section{Discussion}

One of the major particularities of the PVDI-dependent iron acquisition pathway in P. aeruginosa, and probably conserved among fluorescent Pseudomonads, is that this siderophore delivers iron into the bacterial periplasm, with siderophore-free iron then being transported further by an $\mathrm{ABC}$ transporter into the cytoplasm. This mechanism is completely different from that described previously for other siderophore-dependent iron-uptake pathways, such as the enterobactin and ferrichrome pathways in E. coli, two archetypes in the field of bacterial iron homeostasis, which deliver iron directly into the bacterial cytoplasm ${ }^{34}$. After the uptake of PVDI-Fe ${ }^{3+}$ across the outer membrane by FpvAI or $\mathrm{FpvB}^{21-23}$, iron release from PVDI in the bacterial periplasm requires the FpvGHJKCDEF proteins ${ }^{28}$. Moreover, the molecular mechanism involved implies both iron reduction by FpvG reductase to decrease the affinity of PVDI for the metal and an iron chelator, $\mathrm{FpvC}^{26-28}$.

We used a systematic BACTH assay to unravel the interactions between these proteins and highlight specific interactions between FpvG reductase, the inner-membrane protein $\mathrm{FpvH}$, and the three periplasmic proteins FpvJ, FpvC, and FpvF (Fig. 8). Although our BACTH screening was carried out using both N- and C-terminal T18/T25 tags, we observed no interactions with FpvK, suggesting that either (i) FpvK does not interact with the three other proteins, (ii) the interactions are transient or of weak affinity, (iii) the interaction requires a third protein partner, or (iv) the interaction is just not detectable by BACTH. Indeed, fusion to the T18 or T25 domains may affect the folding of the protein or prevent interaction $\mathrm{s}^{35}$.

We biochemically confirmed the interaction between the two inner-membrane proteins FpvG-FpvH by affinity and size-exclusion chromatography, but we still know nothing about the stoichiometry of the FpvG-FpvH complex, except that FpvG is able to form dimers based on the BACTH data. Previously, the in vivo kinetics of iron dissociation from PVDI showed that FpvG activity is dependent on FpvH expression ${ }^{28}$. FpvJ and FpvK expression also affect FpvG activity, but clearly to a lower extent ${ }^{28}$. The ability of FpvK to affect FpvG reductase activity, like FpvH and FpvJ, highly suggests that it also interacts with the other inner-membrane proteins.

BACTH screening also showed an interacting network between the three periplasmic proteins FpvC, FpvF, and FpvJ. This complex was validated by pulldown experiments. $\mathrm{FpvC}$ and $\mathrm{FpvF}$ are two periplasmic-binding proteins associated with the $\mathrm{ABC}$ transporter FpvDE. Purified FpvC was shown to chelate ferrous iron in an in vitro PVDI-Fe dissociation assay using DTT as the iron reducer ${ }^{28}$. Mass spectrometry approaches under native conditions have shown that FpvF can bind PVDI, and that FpvC and FpvF are both able to form the tetrameric $\mathrm{FpvC}_{2}-\mathrm{FpvF}_{2}$-PVDI-Fe complex in the presence of PVDI-Fe ${ }^{25}$. The existence of FpvC-FpvF-PVD-Fe complexes 
A.

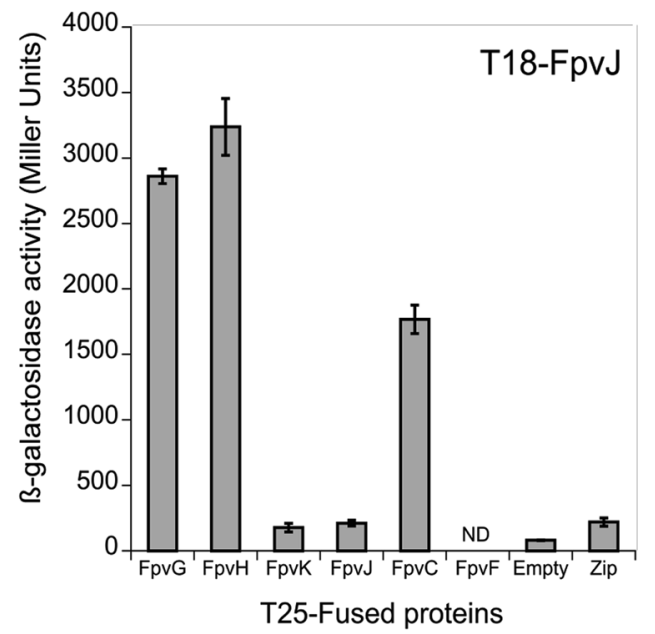

B.

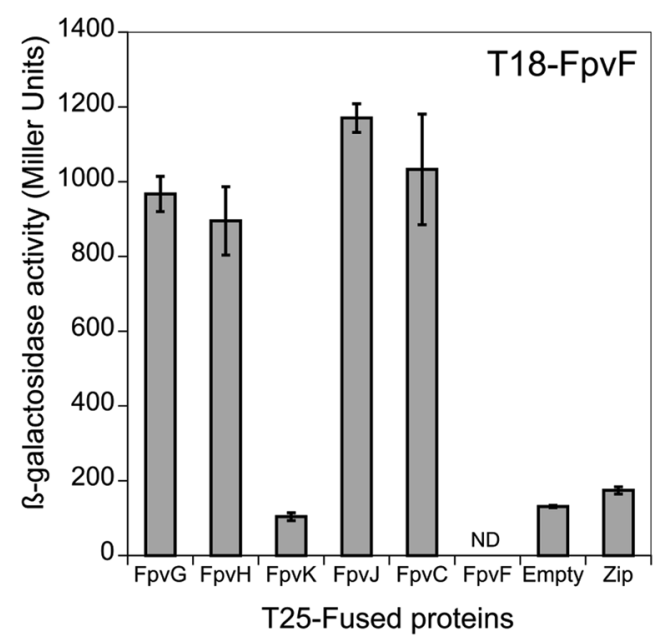

C.

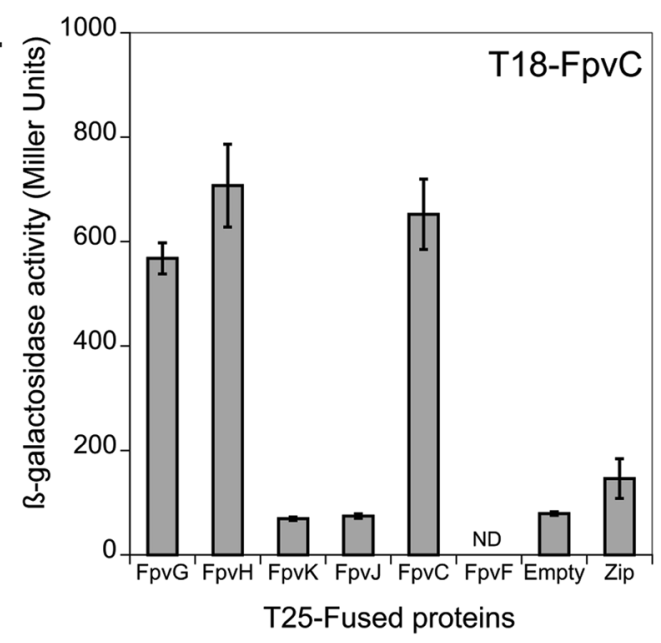

Figure 4. Interacting network with periplasmic (FpvC, FpvF and FpvJ) and membrane proteins (FpvG, FpvH and FpvK). Bacterial two-hybrid assays for proteins were quantified by measuring the $\beta$-galactosidase activity, as described in Materials and Methods. Zip, which is not related to the PVDI pathway, served as a positive control ${ }^{38}$. ND: not determined because for FpvF we were unable to obtain the pKT25-FpvF vector. The experiment was repeated three times independently. Error bars represent the standard errors of the means.

in $P$. aeruginosa periplasm was confirmed by cross-linking experiments ${ }^{25}$, and we proposed that, after its uptake across the outer membrane, PVDI-Fe forms a FpvC-FpvF-PVDI-Fe complex. Isolation by pulldown experiments of the FpvC-FpvF-FpvJ complex strongly suggests that such a complex may exist in the bacterial periplasm of $P$. aeruginos a and is probably necessary in the mechanisms involved in iron release from PVDI. 


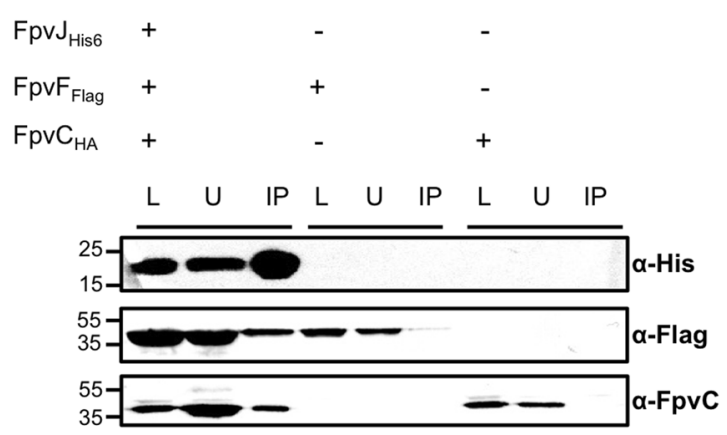

Figure 5. Pulldown assay of the periplasmic FpvJ-FpvF-FpvC complex. Periplasmic fractions of BL21(DE3) cells co-expressing the indicated proteins $\mathrm{FpvJ}_{\mathrm{His}}, \mathrm{FpvC}_{\mathrm{HA}}$, or $\mathrm{FpvF}_{\mathrm{Flag}}$ were incubated with anti-His beads. The loading (L), unbound (U), and immunoprecipitated (IP) fractions were separated by SDS-PAGE and analyzed by immunodetection using anti-His ${ }_{6}$, anti-FpvC and anti-Flag antibodies. The molecular mass markers $(\mathrm{kDa})$ are indicated on the left. The predicted size of $\mathrm{FpvJ}_{\mathrm{His}}, \mathrm{FpvF}_{\mathrm{Flag}}$ and $\mathrm{FpvC}_{\mathrm{HA}}$ are $15 \mathrm{kDa}, 33 \mathrm{kDa}$ and $36 \mathrm{kDa}$ respectively. Entire blots are shown in Fig. S3 in Supplementary Information.

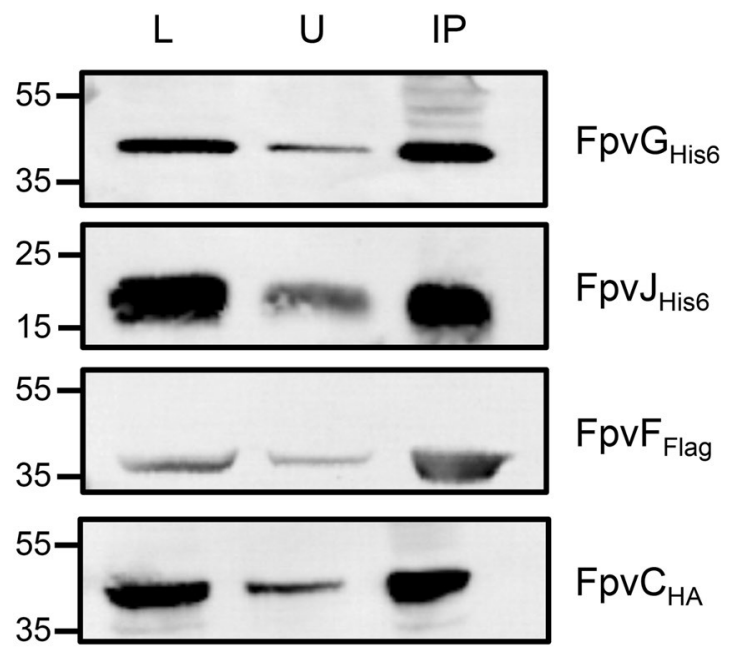

Figure 6. Pulldown assay of the FpvG-FpvJ-FpvC-FpvF complex. Periplasmic fractions of BL21(DE3) cells producing $\mathrm{FpvJ}_{\mathrm{His} 6}, \mathrm{FpvC}_{\mathrm{HA}}, \mathrm{FpvF}_{\mathrm{Flag}}$ and membrane fraction of TOP10 cells producing $\mathrm{FpvG}_{\mathrm{His} 6}$, and $\mathrm{FpvH}_{\text {Strep }}$ were incubated with anti-Flag beads. The loading (L), unbound (U), and immunoprecipitated (IP) fractions were separated by SDS-PAGE and analyzed by immunodetection using anti-His ${ }_{6}$, anti-FpvC, and anti-Flag antibodies. The molecular mass markers $(\mathrm{kDa})$ are indicated on the left. The predicted size are for FpvG $\mathrm{His}_{6}$ $46 \mathrm{kDa}, \mathrm{FpvH}_{\text {Strep }} 21 \mathrm{kDa}, \mathrm{FpvJ}_{\mathrm{His}} 15 \mathrm{kDa}, \mathrm{FpvF}_{\mathrm{Flag}} 33 \mathrm{kDa}$ and $\mathrm{FpvC}_{\mathrm{HA}} 36 \mathrm{kDa}$. Entire blots are shown in Fig. S4 Supplementary Information.

The function of FpvJ is currently unknown, but this protein may allow interaction of the FpvC-FpvF-PVDI-Fe complex with the FpvG-FpvH inner-membrane complex to achieve iron reduction and the transfer of ferrous iron from PVDI to FpvC. BACTH screening showed that all three periplasmic proteins FpvJ, FpvC, and FpvF can interact with the two-protein FpvG-FpvH complex in the absence or presence of PVD-Fe, forming an inner-membrane machinery. We were able to isolate four of the five proteins by pulldown assay, confirming the existence of a complex between the inner-membrane reductase, FpvG, and the three periplasmic proteins, FpvF, FpvC, and FpvJ. Immunodetection of $\mathrm{FpvH}_{\text {Strep }}$ with anti-Strep antibodies revealed non-specific bands of various molecular weights, preventing us from assessing the presence of FpvH in the pulldown assay (data not shown). The exact stoichiometry of this complex is still unknown.

Moreover, FpvDE is the putative ABC transporter that allows the translocation of ferrous iron across the inner membrane into the cytoplasm, and its deletion affects iron acquisition by PVDI $^{25,28}$. We also evaluated the possible interactions of FpvDE with the periplasmic FpvC and FpvF and the membrane proteins FpvG, FpvH, and FpvK, but were unable to detect any interaction (data not shown). However, FpvC probably plays a role in bringing ferrous iron to the permease, $\mathrm{FpvE}$, but further biochemical studies will be necessary to demonstrate this.

Finally, previous studies have demonstrated that FpvF dimers can bind apo-PVDI and apo-PVDI recycling is partially abolished in a $\Delta f p v F$ mutant ${ }^{25,28}$. We thus investigated whether FpvF can interact with the PvdRT-OpmQ efflux pump involved in PVDI recycling. We found that FpvF interacts with PvdT, the inner-membrane protein of the efflux pump. This result strongly supports the hypothesis that $\mathrm{FpvF}_{\mathrm{F}}$ or $\mathrm{FpvF}_{2}$ binds apo-PVDI in a FpvF-PVDI or $\mathrm{FpvF}_{2}-\mathrm{PVDI}_{2}$ complex and brings the apo-siderophore to PvdT for its recycling into the extracellular medium. 


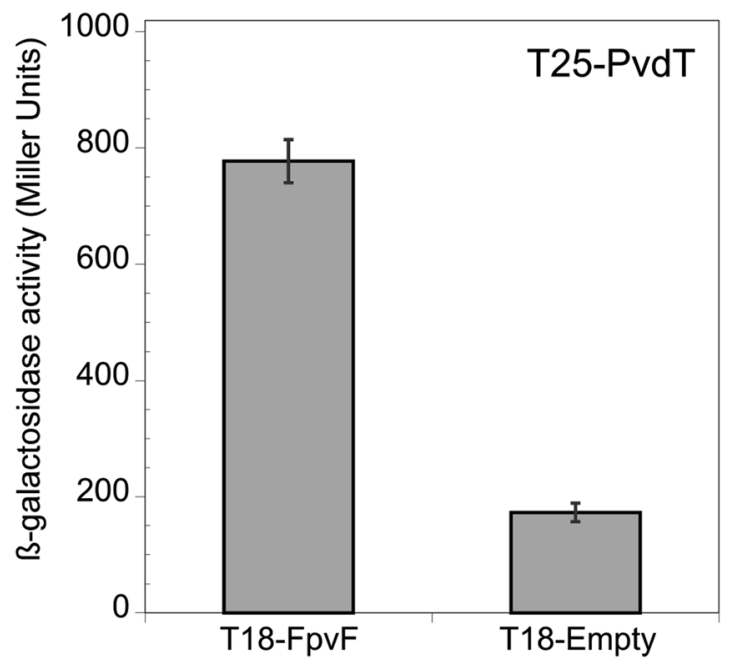

Figure 7. Interaction between the periplasmic FpvF protein and the inner-membrane PvdT protein of the efflux pump PvdRT-OpmQ. Bacterial-two hybrid assays were quantified by measuring the $\beta$-galactosidase activity, as described in Materials and Methods. The experiment was repeated three times independently. Error bars represent the standard errors of the means.

A.

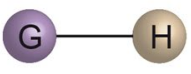

B.

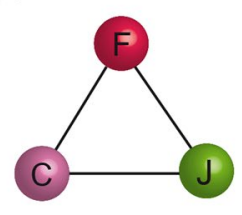

C.

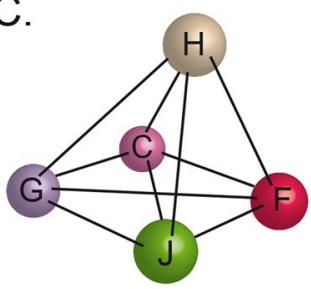

D.

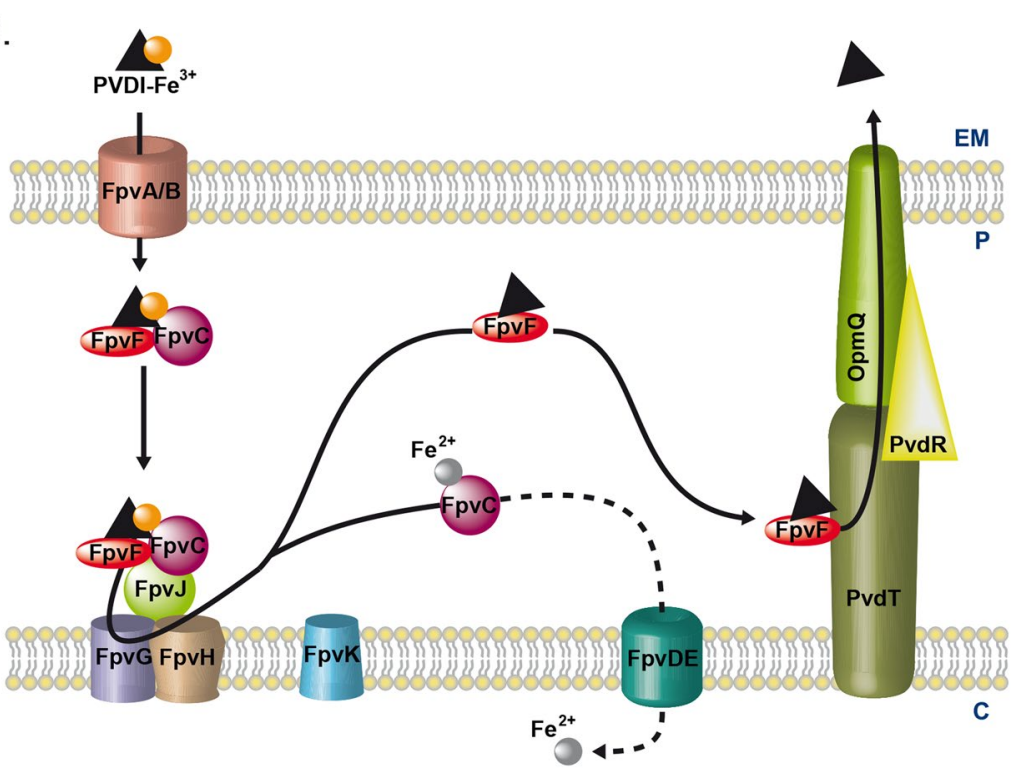

Figure 8. Model of the multi-protein machinery involved in iron acquisition via PVDI in P. aeruginosa. (A) Interacting network observed between inner membrane FpvG and $\mathrm{FpvH}$ proteins. (B) Interacting network between periplasmic proteins FpvF, FpvC and FpvJ. (C) Interacting network between inner membrane and periplasmic proteins. D. Model of PVDI-Fe ${ }^{3+}$ uptake by P. aeruginosa with the protein complexes highlighted. For details, see the Discussion section. EM: extracellular medium; P: periplasm; C: cytoplasm.

In conclusion, this study provides new insights about the possible interacting network of the various proteins involved in iron release from PVDI in the periplasm of $P$. aeruginosa (Fig. 8). These interactions have been highlighted using a two-hybrid approach and confirmed in vitro using purified or pulldown experiments; but 
they existence still need to be confirmed in P. aeruginosa cells. This complex interacting network strongly suggests a multi-protein complex at the inner membrane, allowing iron to be removed from PVDI. Consequently, the molecular mechanisms for iron acquisition via PVDI involves the following steps (Fig. 8D). As detailed in the introduction, PVDI-Fe ${ }^{3+}$ is imported across the outer membrane into the bacterial periplasm by FpvAI and FpvB. In the periplasm, PVDI forms a FpvC-FpvF-PVDI-Fe complex with the two periplasmic-binding proteins. As FpvJ was found to interact with both periplasmic and membrane proteins, it may help in the interaction of this periplasmic complex with the inner-membrane FpvG-FpvH complex. Iron reduction by FpvG decreases the affinity of PVDI for the metal and a transfer of iron to the periplasmic-binding protein FpvC, which likely brings iron to the FpvDE ABC transporter. Apo-PVDI is most likely bound to FpvF, which is able to interact with PvdT, allowing the recycling of apo-PVDI to the extracellular medium by the efflux pump PvdRTOpmQ. Moreover, this FpvGHJCF complex is the first example to be described of a complex between an inner-membrane reductase and two periplasmic-binding proteins associated with an $\mathrm{ABC}$ transporter.

Deciphering and understanding the protein-protein interacting network is an important piece in the understanding of the PVDI-Fe ${ }^{3+}$ uptake pathway puzzle. Our work will undoubtedly initiate a number of future directions like chemical crosslinking experiments in $P$. aeruginosa cells with tagged proteins to assess the existence of this interacting network in the pathogen. Electron microscopy approaches are planned to obtain structural information on these different protein complexes. At last, further studies are also needed to understand the exact role of FpvH, FpvJ, and FpvK within the complexes and PVDI-Fe dissociation.

\section{Materials and Methods}

Chemicals, bacterial strains and growth medium. Medium culture Lysogeny Broth (LB) and LB-agar were purchased from Difco. Detergent n-dodecyl- $B$-D-maltoside (DDM) was purchased from Anatrace, $\mathrm{N}$-Lauroylsarcosine sodium (SLS) and Tween 20 from Sigma. The strains used in this study are listed in the Supplementary Table S1. Briefly, TOP10 and DH5 $\alpha$ strains were used for cloning procedures, TOP10 and BL21 strains for protein production and DHM1 strains for bacterial two-hybrid assays. E. coli strains were routinely grown in LB medium at $37^{\circ} \mathrm{C}$ and on LB-agar for solid culture. Plasmids were maintained by the addition of antibiotics such as ampicillin $(100 \mu \mathrm{g} / \mathrm{ml})$, kanamycin $(50 \mu \mathrm{g} / \mathrm{ml})$, chloramphenicol $(50 \mu \mathrm{g} / \mathrm{ml})$ and streptomycin $(100 \mu \mathrm{g} / \mathrm{ml})$.

Plasmid construction. Plasmids used in this study are listed in Supplementary Table S1. All the PCRs were performed with DNA Phusion high-fidelity polymerase (Thermofischer Scientific). DNA sequences from Pseudomonas aeruginosa PAO1 were taken from Pseudomonas Genome DataBank (www.pseudomonas.com). Oligonucleotides were purchased from Sigma and are listed in Supplementary Table S2. All primers used introduced restriction sites.

Bacterial two-hybrid vectors. pKT25 and pUT18C vectors were used for the expression of membrane proteins, whereas pKTM25-zip and pUTM18C vectors were used for the periplasmic proteins. Coding regions of $f p v G$, $f p v H, f p v K, f p v E, f p v D$ and $p v d T$ were amplified by PCR with primer pairs $1159 / 1160,1161 / 1162,1177 / 1178$, $1199 / 1200,1197 / 1198$ and 1420/1411 respectively. The PCR products were digested with KpnI/XbaI and inserted into the same sites of pKT25 or pUT18C vectors to create pKT25-FpvG, pKT25-FpvH, pKT25-FpvK, pKT25-FpvD, pKT25-FpvE, pUT18C-FpvG, pUT18C-FpvH, pUT18C-FpvK, pUT18C-FpvD, pUT18C-FpvE and pKT25-PvdT. Coding regions of $f p v J$ and $f p v C$ were amplified with primer pairs 1397/1201 and 1408/1202 respectively, and the relevant PCR products were digested with XbaI/KpnI or XbaI/SacI and cloned into the same sites of pKTM25-zip to create pKTM25-FpvJ and pKTM25-FpvC. To clone into the pUTM18C vector, $f p v J, f p v C$ and $f p v F$ genes were amplified with primer pairs 1397/1212, 1408/1400 and 1399/1203 respectively, and then digested with XbaI/KpnI to be inserted into the same restriction sites of pUTM18C to create pUTM18C-FpvJ, pUTM18C-FpvC and pUTM18C-FpvF.

Protein production vectors. To construct $\mathrm{pBAD} 24-\mathrm{FpvG}_{\mathrm{His} 6}$ and $\mathrm{pBAD} 33-\mathrm{FpvH}_{\text {Strep }}$ vectors, coding regions of $f p v G$ and $f p v H$ were amplified with primer pairs $1185 / 1186$ and $1189 / 1190$, respectively. PCR products of $f p v G$ and $f p v H$ were digested with NcoI/HindIII and EcoRI/HindIII and inserted into the same sites of pBAD24 or pBAD33. To clone into the pRSF and pCDF vectors, primer pairs 1404/1405, 1408/1409 and 1406/1407 were used to amplify $f p v J, f p v C$ and $f p v F$, respectively. PCR products were digested by EcoRI/HindIII for $f p v J$ and $f p v C$, and $B g l I I / K p n I$ for $f p v F$ and were cloned into the same restriction sites of pRSF or pCDF vectors to create pCDF-FpvJ $\mathrm{His}_{\mathrm{H} 6}, \mathrm{pCDF}-\mathrm{Fpv} \mathrm{H}_{\mathrm{His} 6} \mathrm{FpvF}_{\mathrm{Flag}}, \mathrm{pRSF}-\mathrm{FpvC}_{\mathrm{HA}}$ and $\mathrm{pCDF}-\mathrm{FpvF}_{\mathrm{Flag}}$.

All constructs were screened with colony PCR and plasmids were purified with the Macherey Nagel Nucleospin Plasmid kit in accordance with the manufacturer's instructions. All constructions were verified by DNA sequencing (Eurofins).

Bacterial two-hybrid assay. For plate-BACTH assay, two compatible vectors producing proteins fused to $\mathrm{T} 18$ or T25 domain were co-transformed into DHM1 cells that were incubated at $30^{\circ} \mathrm{C}$ for $16 \mathrm{~h}$. Ten independent colonies of each transformation were inoculated together into $2 \mathrm{ml}$ of LB medium supplemented with ampicillin, kanamycin and $0.5 \mathrm{mM}$ isopropyl $\beta$-D-1-thiogalactopyranoside (IPTG, Sigma) and incubated at $30^{\circ} \mathrm{C}$ for $16 \mathrm{~h}$. $5 \mu \mathrm{l}$ of each culture was spotted onto LB-agar plate supplemented with appropriate antibiotics, $0.5 \mathrm{mM}$ IPTG and $40 \mu \mathrm{g} / \mathrm{ml}$ 5-bromo-4-chloro-3-indolyl- $\beta$-D-galactopyranoside (X-gal, Sigma). The plate was incubated for $16 \mathrm{~h}$ at $30^{\circ} \mathrm{C}$.

For liquid medium assay, two compatible vectors producing proteins fused to T18 or T25 domain were co-transformed into DHM1 cells that were incubated at $37^{\circ} \mathrm{C}$ for $16 \mathrm{~h}$. Ten independent colonies of each transformation were inoculated into $2 \mathrm{ml}$ of LB-medium supplemented with appropriate antibiotics and were 
incubated at $37^{\circ} \mathrm{C}$ during $24 \mathrm{~h}$. The next day, $20 \mu \mathrm{l}$ of each culture were inoculated in $2 \mathrm{ml}$ of $\mathrm{LB}$ supplemented with appropriate antibiotics and $0.5 \mathrm{mM} \mathrm{IPTG}$ and incubated at $37^{\circ} \mathrm{C}$ for $16 \mathrm{~h} .100 \mu \mathrm{l}$ of each culture was used for the $ß$-galactosidase assay using Miller Protocol ${ }^{36}$.

ß-galactosidase dosage. $\quad 100 \mu \mathrm{l}$ of bacterial culture were added to $900 \mu \mathrm{l}$ of $\mathrm{Z}$ Buffer $\left(60 \mathrm{mM} \mathrm{Na}_{2} \mathrm{HPO}_{4}\right.$, $40 \mathrm{mM} \mathrm{NaH}_{2} \mathrm{PO}_{4}, 10 \mathrm{mM} \mathrm{KCl}, 1 \mathrm{mM} \mathrm{MgSO}_{4}, \mathrm{pH} 7.0,0.2 \% \beta$-mercaptoethanol). $1 \mu \mathrm{l}$ of $0.1 \%$ sodium dodecylsulfate and $50 \mu \mathrm{l}$ of chloroform were added to the suspension that was mixed vigorously for 10 seconds. The suspension was then incubated for $5 \mathrm{~min}$ at $28^{\circ} \mathrm{C}$. $200 \mu \mathrm{l} \mathrm{of} 4 \mathrm{mg} / \mathrm{ml}$ 2-nitrophenyl B-D-galactopyranoside (ONPG, Sigma) were added to the cells. Reaction was stopped by adding $500 \mu \mathrm{l}$ of $1 \mathrm{M} \mathrm{Na}_{2} \mathrm{CO}_{3}$. The suspension was centrifuged at $14,000 \mathrm{~g}$ for $3 \mathrm{~min}$ and the optical density of the supernatant was read at 420 and $550 \mathrm{~nm}$. The ß-galactosidase activity was then calculated in Miller Unit (MU) according to the following equation:

$$
\mathrm{MU}=\frac{1000 \times\left(\mathrm{OD}_{420 \mathrm{~nm}}-1.75 \times \mathrm{OD}_{550 \mathrm{~nm}}\right)}{\text { Time }(\mathrm{min}) \times \operatorname{Volume}(\mathrm{mL}) \times \mathrm{OD}_{600 \mathrm{~nm}}}
$$

Expression and purification of the FpvG-FpvH inner membrane complex. E. coli TOP10 strain was co-transformed with pBAD24-FpvG $\mathrm{His}_{6}$ and $\mathrm{pBAD} 33-\mathrm{FpvH}_{\text {Strep }}$. Overnight culture was inoculated into LB medium supplemented with ampicillin and chloramphenicol and grown at $37^{\circ} \mathrm{C}$ until $\mathrm{OD}_{600 \mathrm{~nm}}$ reached 0.6 . Then, protein production was induced by addition of $0.01 \% \mathrm{~L}$-arabinose for $4 \mathrm{~h}$ at $30^{\circ} \mathrm{C}$. Cell pellet was re-suspended in $50 \mathrm{mM}$ Tris- $\mathrm{HCl} \mathrm{pH} \mathrm{8.0,100} \mathrm{mM} \mathrm{NaCl}$ and one protease inhibitor tablet (complete EDTA-Free protease inhibitor, Roche). After sonication, unbroken cells were removed by centrifugation at $12,000 \mathrm{~g}$ for $15 \mathrm{~min}$ at $4{ }^{\circ} \mathrm{C}$. The supernatant was ultracentrifuged during $40 \mathrm{~min}$ at $100,000 \mathrm{~g}$ at $4^{\circ} \mathrm{C}$. The pellet was re-suspended into $50 \mathrm{mM}$ Tris- $\mathrm{HCl}$ $\mathrm{pH} 8.0,50 \mathrm{mM} \mathrm{NaCl}, 1 \% \mathrm{DDM}$ and solubilized overnight at $4^{\circ} \mathrm{C}$. Membranes were collected by ultracentrifugation at $100,000 \mathrm{~g}$ for $40 \mathrm{~min}$ at $4^{\circ} \mathrm{C}$ and loaded onto a StrepTrap Column (GE Healthcare) equilibrated with Buffer A (50 mM Tris- $\mathrm{HCl} \mathrm{pH} \mathrm{8.0,} 50 \mathrm{mM} \mathrm{NaCl}, 0.1 \%$ DDM). The FpvG-FpvH complex was eluted with Buffer A supplemented with $2.5 \mathrm{mM}$ D-Desthiobiotin (Sigma). Fractions of interest were then concentrated using a $10,000 \mathrm{kDa}$ molecular weight cut off (Amicon, Millipore) and loaded onto a Superdex 200 10/300 GL column equilibrated with $50 \mathrm{mM}$ Tris- $\mathrm{HCl} \mathrm{pH}$ 8.0, $50 \mathrm{mM} \mathrm{NaCl}, 0.025 \%$ DDM. Fractions containing $\mathrm{FpvH}_{\text {Strep }}$ and FpvG $\mathrm{His}_{6}$ were collected for further analysis.

Expression of the FpvJ-FpvF-FpvC periplasmic complex. BL21(DE3) cells were transformed with pCDF-Fpv $\mathrm{His}-\mathrm{FpvF}_{\mathrm{Flag}}$ and $\mathrm{pRSF}-\mathrm{FpvC}_{\mathrm{HA}}$. Overnight culture was inoculated into LB medium with streptomycin and kanamycin and grown at $37^{\circ} \mathrm{C}$ until $\mathrm{OD}_{600}$ reached 0.6 . Then, protein production was induced by adding $0.4 \mathrm{mM}$ IPTG at $22^{\circ} \mathrm{C}$ for $16 \mathrm{~h}$. Cells were subjected to cellular fractionation as described below for further analysis.

Immunoblot analysis. Nitrocellulose membranes were used for protein transfer by electroblotting. After saturation with blocking buffer (5\% dried-milk powder, PBS 1X, 0.1\% Tween 20), the membranes were incubated overnight at $4{ }^{\circ} \mathrm{C}$ with primary antibody: anti-Strep (1/2000, IBA), anti-HA (1/2000, Sigma), anti-FpvC (laboratory collection), anti-His ${ }_{6}(1 / 3000$, GeneTex), anti-OmpC (Biorbyt), anti-LepB (Biorbyt), anti-Ef-Tu (LSBio) or anti-MBP (Bioss antibodies). Immunoblots were developed by using horseradish peroxidase-conjugated goat anti-rabbit or anti-mouse antibodies (1/10000, GE Healthcare) followed by chemiluminescence detection. Molecular mass marker was purchased from Thermofischer Scientific.

Cellular fractionation. Periplasm and cytoplasm isolation. E. coli strains overproducing proteins of interest were pelleted, washed with $50 \mathrm{mM}$ Tris- $\mathrm{HCl} \mathrm{pH} 8.0$ and re-suspended into Tris-Sucrose EDTA Buffer (0.2 M Tris- $\mathrm{HCl} \mathrm{pH} \mathrm{8.0,20 \%} \mathrm{sucrose} \mathrm{and} 1 \mathrm{mM}$ EDTA). $200 \mu \mathrm{g} / \mathrm{ml}$ of lysozyme were added to the suspension, incubated for $1 \mathrm{~h}$ at $4{ }^{\circ} \mathrm{C}$ and the cells were centrifuged at $6,700 \mathrm{~g}$ for $10 \mathrm{~min}$ at $4{ }^{\circ} \mathrm{C}$ to remove unbroken cells and insoluble fraction (like insoluble proteins). The supernatant corresponding to the periplasm was ultracentrifuged at $100,000 \mathrm{~g}$ for $40 \mathrm{~min}$ at $4^{\circ} \mathrm{C}$. Spheroplasts were washed three times with Tris-Sucrose buffer and re-suspended into chill water and treated with benzonase (Sigma, 250U/ $\mu \mathrm{l}$ ). After incubation for $1 \mathrm{~h}$ at $37^{\circ} \mathrm{C}$, suspension was centrifuged for $40 \mathrm{~min}$ at $100,000 \mathrm{~g}$ at $4^{\circ} \mathrm{C}$ to collect the cytoplasm (supernatant). The pellet, corresponding to the total membranes, was re-suspended in $50 \mathrm{mM}$ Tris- $\mathrm{HCl} \mathrm{pH} 8.0$.

Membrane isolation. E. coli strains overproducing proteins of interest were pelleted, re-suspended in $50 \mathrm{mM}$ Tris- $\mathrm{HCl} \mathrm{pH} 8.0$ and lysed by sonication. Unbroken cells were removed by centrifugation at $12,000 \mathrm{~g}$ for $15 \mathrm{~min}$. Supernatant was centrifuged at $100,000 \mathrm{~g}$ for $40 \mathrm{~min}$. The membranes (pellet) were solubilized in $50 \mathrm{mM}$ Tris $\mathrm{pH}$ $8.0,100 \mathrm{mM} \mathrm{NaCl}, 0.1 \% \mathrm{SLS}$ for $16 \mathrm{~h}$ at $4^{\circ} \mathrm{C}$ and ultracentrifuged at $100,000 \mathrm{~g}$ for $40 \mathrm{~min}$ at $4{ }^{\circ} \mathrm{C}$. The pellet corresponds to the outer membranes and the supernatant to the inner membranes.

Pulldown experiments. Periplasmic proteins. BL21 (DE3) cells were transformed with pCDF-Fpv $\mathrm{His}-\mathrm{FpvF}_{\mathrm{Flag}}$ and $\mathrm{pRSF}-\mathrm{FpvC}_{\mathrm{HA}}$ or with $\mathrm{pCDF}-\mathrm{FpvF}_{\mathrm{Flag}}$ or pRSF-FpvC $\mathrm{C}_{\mathrm{HA}}$ only. Overnight culture was inoculated into $\mathrm{LB}$ medium with appropriate antibiotics and grown at $37^{\circ} \mathrm{C}$ until $\mathrm{OD}_{600}$ reached 0.6 . Then protein production was induced by adding $0.4 \mathrm{mM}$ IPTG at $22^{\circ} \mathrm{C}$ for $16 \mathrm{~h}$. Cells were re-suspended in buffer A $(50 \mathrm{mM}$ Tris pH 8.0, $250 \mu \mathrm{M}$ EDTA, 20\% sucrose), subjected to cellular fractionation and the periplasmic fraction was recovered. $100 \mu \mathrm{l}$ of the periplasmic fraction were mixed with $50 \mu \mathrm{l}$ agarose beads charged with nickel (Sigma) and incubated on a rotating wheel for $1 \mathrm{~h}$ at room temperature. The mixture was then centrifuged $2 \mathrm{~min}$ at $2,000 \mathrm{rpm}$ 
to remove the unbound proteins. Beads were washed twice with $50 \mathrm{mM}$ Hepes pH 7.5, $50 \mathrm{mM} \mathrm{NaCl}$ before being recovered by centrifugation for further analysis.

Periplasmic and membrane proteins. Periplasmic fractions containing $\mathrm{Fpv}_{\mathrm{His} 6}, \mathrm{FpvF}_{\mathrm{Flag}}, \mathrm{FpvC}_{\mathrm{HA}}$ were incubated with solubilized membranes isolated from TOP10 cells producing $\mathrm{FpvG}_{\mathrm{His} 6}$ and $\mathrm{FpvH}_{\text {Strep }}$ proteins and submitted to anti-Flag resin (Sigma). The next steps were performed as described above. When the experiment has been carried out in the presence of PVDI-Fe, $10 \mu \mathrm{M}$ of the ferri-siderophore complex have been added during incubation with the anti-Flag resin.

Received: 5 June 2019; Accepted: 18 December 2019;

Published online: 10 January 2020

\section{References}

1. Tommasi, R., Brown, D. G., Walkup, G. K., Manchester, J. I. \& Miller, A. A. ESKAPEing the labyrinth of antibacterial discovery. Nat Rev Drug Discov 14, 529-542 (2015).

2. Lyczak, J. B., Cannon, C. L. \& Pier, G. B. Establishment of Pseudomonas aeruginosa infection: lessons from a versatile opportunist. Microbes and infection/Institut Pasteur 2, 1051-60 (2000).

3. Lamont, I. L., Beare, P. A., Ochsner, U., Vasil, A. I. \& Vasil, M. L. Siderophore-mediated signaling regulates virulence factor production in Pseudomonas aeruginosa. Proc Natl Acad Sci USA 99, 7072-7 (2002).

4. Hider, R. Siderophore mediated absorption of iron. Struct Bonding 58, 28 (1984).

5. Meyer, J. M., Neely, A., Stintzi, A., Georges, C. \& Holder, I. A. Pyoverdin is essential for virulence of Pseudomonas aeruginosa. Infect Immun 64, 518-23 (1996).

6. Xiong, Y. Q., Vasil, M. L., Johnson, Z., Ochsner, U. A. \& Bayer, A. S. The oxygen- and iron-dependent sigma factor pvdS of Pseudomonas aeruginosa is an important virulence factor in experimental infective endocarditis. J. Infect. Dis. 181, 1020-1026 (2000).

7. Imperi, F. et al. Repurposing the antimycotic drug flucytosine for suppression of Pseudomonas aeruginosa pathogenicity. Proc. Natl. Acad. Sci. USA 110, 7458-7463 (2013).

8. Minandri, F. et al. Role of Iron Uptake Systems in Pseudomonas aeruginosa Virulence and Airway Infection. Infect. Immun. 84, 2324-2335 (2016).

9. Wilderman, P. J. et al. Characterization of an endoprotease (PrpL) encoded by a PvdS-regulated gene in Pseudomonas aeruginosa. Infect Immun 69, 5385-94 (2001).

10. Demange, P. et al. Bacterial siderophores: structure and NMR assigment of pyoverdins PaA, siderophores of Pseudomonas aeruginosa ATCC 15692. Biol. Metals 3, 155-170 (1990).

11. Budzikiewicz, H. Siderophores of fluorescent pseudomonads. Z Naturforsch [C] 52, 713-20 (1997)

12. Budzikiewicz, H. Siderophores of the Pseudomonadaceae sensu stricto (fluorescent and non-fluorescent Pseudomonas spp.). Fortschritte der Chemie organischer Naturstoffe. Progress in the chemistry of organic natural products 87, 81-237 (2004).

13. Budzikiewicz, H., Schafer, M., Fernandez, D. U., Matthijs, S. \& Cornelis, P. Characterization of the chromophores of pyoverdins and related siderophores by electrospray tandem mass spectrometry. Biometals 20, 135-44 (2007).

14. Fuchs, R., Schafer, M., Geoffroy, V. \& Meyer, J. M. Siderotyping-a powerful tool for the characterization of pyoverdines. Curr Top Med Chem 1, 31-57 (2001).

15. Meyer, J. M. et al. Use of siderophores to type pseudomonads: the three Pseudomonas aeruginosa pyoverdine systems. Microbiology 143(Pt 1), 35-43 (1997)

16. Visca, P., Imperi, F. \& Lamont, I. L. Pyoverdine siderophores: from biogenesis to biosignificance. Trends in microbiology 15, 22-30 (2007).

17. Gasser, V., Guillon, L., Cunrath, O. \& Schalk, I. J. Cellular organization of siderophore biosynthesis in Pseudomonas aeruginosa: Evidence for siderosomes. Journal of inorganic biochemistry 148, 27-34 (2015).

18. Yeterian, E. et al. Synthesis of the siderophore pyoverdine in Pseudomonas aeruginosa involves a periplasmic maturation. Amino Acids 38, 1447-59 (2010).

19. Hannauer, M., Yeterian, E., Martin, L. W., Lamont, I. L. \& Schalk, I. J. Secretion of newly synthesized pyoverdine by Pseudomonas aeruginosa involves an efflux pump. FEBS Lett 584, 4751-5 (2010).

20. Albrecht-Gary, A. M., Blanc, S., Rochel, N., Ocacktan, A. Z. \& Abdallah, M. A. Bacterial iron transport: coordination properties of pyoverdin PaA, a peptidic siderophore of Pseudomonas aeruginosa. Inorg. Chem. 33, 6391-6402 (1994).

21. Poole, K., Neshat, S., Krebes, K. \& Heinrichs, D. E. Cloning and nucleotide sequence analysis of the ferripyoverdine receptor gene fpvA of Pseudomonas aeruginosa. J Bacteriol 175, 4597-604 (1993).

22. Ghysels, B. et al. FpvB, an alternative type I ferripyoverdine receptor of Pseudomonas aeruginosa. Microbiology 150, 1671-80 (2004).

23. Brillet, K. et al. A $\beta$-strand lock-exchange for signal transduction in TonB-dependent transducers on the basis of a common structural motif. Structure 15, 1383-91 (2007).

24. Poole, K., Neshat, S., Krebes, K. \& Heinrichs, D. E. Cloning and nucleotide sequence analysis of the ferripyoverdine receptor gene $f p v A$ of Pseudomonas aeruginosa. Journal of Bacteriology 175, 4597-4604 (1993).

25. Brillet, K. et al. An ABC transporter with two periplasmic binding proteins involved in iron acquisition in Pseudomonas aeruginosa. ACS chemical biology 7, 2036-45 (2012).

26. Schalk, I. J., Abdallah, M. A. \& Pattus, F. Recycling of Pyoverdin on the FpvA Receptor after Ferric Pyoverdin Uptake and Dissociation in Pseudomonas aeruginosa. Biochemistry 41, 1663-1671 (2002).

27. Greenwald, J. et al. Real-time FRET visualization of ferric-pyoverdine uptake in Pseudomonas aeruginosa: a role for ferrous iron. J Biol Chem 282, 2987-2995 (2007).

28. Ganne, G. et al. Iron Release from the Siderophore Pyoverdine in Pseudomonas aeruginosa Involves Three New Actors: FpvC, FpvG, and FpvH. ACS Chem. Biol. 12, 1056-1065 (2017).

29. Imperi, F., Tiburzi, F. \& Visca, P. Molecular basis of pyoverdine siderophore recycling in Pseudomonas aeruginosa. Proceedings of the National Academy of Sciences of the United States of America 106, 20440-5 (2009).

30. Yeterian, E., Martin, L. W., Lamont, I. L. \& Schalk, I. J. An efflux pump is required for siderophore recycling by Pseudomonas aeruginosa. Environ Microbiol Report 2, 412-418 (2010).

31. Gao, L. et al. The Two-Operon-Coded ABC Transporter Complex FpvWXYZCDEF is Required for Pseudomonas aeruginosa Growth and Virulence Under Iron-Limiting Conditions. J. Membr. Biol. 251, 91-104 (2018).

32. Teichmann, S. A. \& Babu, M. M. Conservation of gene co-regulation in prokaryotes and eukaryotes. Trends Biotechnol. 20, 407-410; discussion 410 (2002).

33. Perraud, Q. et al. A Key Role for the Periplasmic PfeE Esterase in Iron Acquisition via the Siderophore Enterobactin in Pseudomonas aeruginosa. ACS Chem. Biol. 13, 2603-2614 (2018).

34. Schalk, I. J. \& Guillon, L. Fate of ferrisiderophores after import across bacterial outer membranes: different iron release strategies are observed in the cytoplasm or periplasm depending on the siderophore pathways. Amino Acids 44, 1267-1277 (2013). 
35. Battesti, A. \& Bouveret, E. The bacterial two-hybrid system based on adenylate cyclase reconstitution in Escherichia coli. Methods 58, 325-334 (2012).

36. Miller, J. H. Experiments in molecular genetics. (Cold Spring Harbor Laboratory, 1972).

37. Vincent, F. et al. Distinct oligomeric forms of the Pseudomonas aeruginosa RetS sensor domain modulate accessibility to the ligand binding site. Environ. Microbiol. 12, 1775-1786 (2010).

38. Ouellette, S. P., Gauliard, E., Antosová, Z. \& Ladant, D. A Gateway $\left({ }^{\circledR}\right)$-compatible bacterial adenylate cyclase-based two-hybrid system. Environ Microbiol Rep 6, 259-267 (2014).

\section{Acknowledgements}

Authors acknowledge the Centre National de la Recherche Scientifique (CNRS) and the University of Strasbourg for general financial support. We would like to thank Roche Pharmaceutical Research and Early Development Basel for their financial support via the Roche Postdoctoral Fellowship (RPF) Program. BR held a fellowship from Roche Postdoctoral Fellowship and AB from the French Ministère de la Recherche et de la Technologie (3 years). We thank Christophe Bordi (LISM, Marseille) and Daniel Ladant (Institut Pasteur, Paris) for kindly providing the two-hybrid vectors.

\section{Author contributions}

Anne Bonneau: Investigation, Writing- Original draft preparation. Béatrice Roche: Conceptualization, Methodology, Writing- Original draft preparation, Supervision, Validation. Isabelle J. Schalk: Conceptualization, Validation, Writing - Review \& Editing, Supervision, Project Administration, Funding Acquisition.

\section{Competing interests}

The authors declare no competing interests.

\section{Additional information}

Supplementary information is available for this paper at https://doi.org/10.1038/s41598-019-56913-X.

Correspondence and requests for materials should be addressed to B.R. or I.J.S.

Reprints and permissions information is available at www.nature.com/reprints.

Publisher's note Springer Nature remains neutral with regard to jurisdictional claims in published maps and institutional affiliations.

Open Access This article is licensed under a Creative Commons Attribution 4.0 International License, which permits use, sharing, adaptation, distribution and reproduction in any medium or format, as long as you give appropriate credit to the original author(s) and the source, provide a link to the Creative Commons license, and indicate if changes were made. The images or other third party material in this article are included in the article's Creative Commons license, unless indicated otherwise in a credit line to the material. If material is not included in the article's Creative Commons license and your intended use is not permitted by statutory regulation or exceeds the permitted use, you will need to obtain permission directly from the copyright holder. To view a copy of this license, visit http://creativecommons.org/licenses/by/4.0/.

(c) The Author(s) 2020 